\title{
Identification of Proliferative Diabetic Retinopathy Using Texture Segmentation
}

\author{
${ }^{1}$ Tamil Pavai, G. and ${ }^{2}$ S. Tamil Selvi \\ ${ }^{1}$ Department of Computer Science and Engineering, \\ Government College of Engineering, Tirunelveli, Tamil Nadu, 627007, India \\ ${ }^{2}$ Department of Electronics and Communication, \\ National Engineering College, Kovilpatti, Tamil Nadu, 628501, India
}

Received 2013-03-13, Revised 2013-04-18; Accepted 2013-04-26

\begin{abstract}
In this study, it is proposed fast texture segmentation of detection of proliferative diabetic retinopathy. proliferative diabetic retinopathy is characterized by the revascularization on the optic disc or on the retinal area or both, as a result of diabetes mellitus. It may leads to the blindness if it is not detected and treated in proper time. This study proposes a novel method to detect the vessel growth on the optic disc using texture segmentation. The vessels that grow on the optic disc as a result of proliferative diabetic retinopathy will be more convoluted than the normal vessel. The segmentation of the optic disc is made using the texture descriptors. Next, the segmentation of blood vessel is made by Ridge strength and Watershed Transformation. Fifteen features which represent the shape, gradient of the vessel are calculated. The calculated features are separated using a hyperplane to classify it as normal or abnormal by support vector machine classifier. We have observed a sensitivity and specificity of 88.89 and $91.3 \%$.
\end{abstract}

Keywords: Texture Descriptors, Support Vector Machine, Tortuosity Measure, Vasculature, Rigid Strength and Watershed Transformation

\section{INTRODUCTION}

Texture is an important factor in visual perception and discrimination of image content. Texture segmentation plays a vital role in texture analysis. Even though texture analysis is a four decade research issue, it still having lot of problem to be solved and automated. Texture analysis falls into three major category, texture classification, texture segmentation and shape from texture (Chen et al., 1999). Texture segmentation is highly useful in medical analysis, defect detection in images of textiles and automated inspection of carpet wear and automobile paints, document processing and in remote sensing identification of landmarks, such as mountains and lakes in Ariel photography. In medical analysis, texture features are powerful in getting the nature of diseases.
Medical fields such as pathology, dermatology and ophthalmology can be under gone texture segmentation methods to determine either the nature of dieses or the seriousness of the dieses. Texture segmentation is to segment an image into regions according to the textures of the regions shown in Fig. 1. Texture segmentation majorly classified into supervised and unsupervised. In the supervised texture segmentation, it is assumed that all the parameters for the textures and for the noise if it is present, are specified and the segmentation is to partition the image in terms of the textures whose distribution functions have been completely specified. Unsupervised segmentation not only does the partition but also needs to estimate the involved parameters obviously; this is more practical in applications and is also more challenging.

Corresponding Author: Tamil Pavai, G., Department of Computer Science and Engineering, Government College of Engineering, Tirunelveli, Tamil Nadu, 627007, India Tel: +91-09442523888 


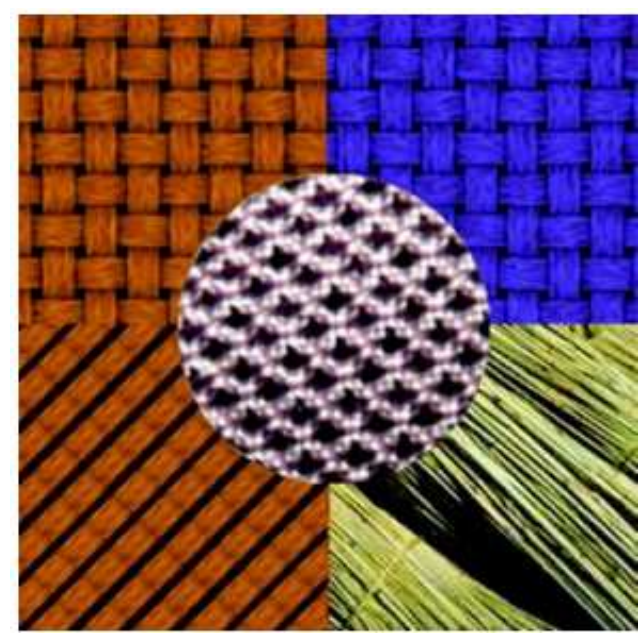

Fig. 1. Texture mosaic

One of the applications in texture segmentation is Diabetic Retinopathy (DR). Diabetic retinopathy is a complication of diabetes and a leading cause of blindness. It occurs when diabetes damages the tiny blood vessels inside the retina, the light-sensitive tissue at the back of the eye. A healthy retina is necessary for good vision. If the patient has diabetic retinopathy, at first they may notice no changes in their vision. But over time, diabetic retinopathy can get worse and cause vision loss. Diabetic retinopathy usually affects both eyes. Diabetic retinopathy has four stages: (1) Mild No proliferative Retinopathy, at this earliest stage, micro aneurysms occur. They are small areas of balloon-like swelling in the retina's tiny blood vessels. (2) Moderate No proliferative Retinopathy, as the disease progresses, some blood vessels that nourish the retina are blocked. (3) Severe No proliferative Retinopathy, many more blood vessels are blocked, depriving several areas of the retina with their blood supply. These areas of the retina send signals to the body to grow new blood vessels for nourishment. (4) Proliferative Retinopathy, at this advanced stage, the signals sent by the retina for nourishment trigger the growth of new blood vessels. This condition is called proliferative retinopathy. These new blood vessels are abnormal and fragile. They grow along the retina and along the surface of the clear, vitreous gel that fills the inside of the eye. If left untreated, diabetic retinopathy can cause blindness.

Proliferative Diabetic Retinopathy (PDR) mainly occurs when many of the blood vessels in the retina close, preventing enough blood flow. In an attempt to supply blood to the area where the original vessels closed, the retina responds by growing new blood vessels. This is called as neovascularization. These new blood vessels are abnormal and do not supply the retina with proper blood flow. The new vessels are also accompanied by scar tissue that may cause the retina to wrinkle or detach. New vessels are classified according to their position as new vessels on the optic disc and new vessels elsewhere. New blood vessels have a narrower calibre, more tortuous and are convoluted than normal vessels. The development of the new vessels can be inhibited by early diagnosis and treatment. There are three major treatments for diabetic retinopathy, which are very effective in reducing vision loss. These three treatments are laser surgery, injection of triamcinolone into the eye and vitrectomy. Number of studies has investigated automatic detection of micro aneurysms or haemorrhages and exudates. Little work has been done to detect vessel abnormalities such as venous beading and vessel characteristics to predict the presence of proliferative disease. Hipwell et al. (2000) proposes an automatic approach to detect diabetic retinopathy in digital red-free images that uses an algorithm that enhances small round features (Hipwell et al., 2000). Walter et al. (2007) proposed the method for the automatic detection of micro aneurysms by diameter closing and an automatic threshold scheme (Walter et al., 2007). proposed a method that detect exudates using their high grey level variation and their contours are determined by means of morphological reconstruction techniques, the optic disc is detected by means of morphological filtering techniques and the watershed transformation (Walter et al., 2002). Osareh et al. (2003) proposed a method to identify exudates using fuzzy Cmeans clustering following some key preprocessing steps. To classify the segmented regions into exudates and nonexudates, an artificial neural network classifier was used (Osareh et al., 2003). Yang et al. (1995) present a computer-aided diagnostic system to automatically detect venous beading of blood vessels. It comprises of two modules, referred to as the Blood Vessel Extraction Module (BVEM) and the Venous Beading Detection Module (VBDM). The former uses a bell-shaped Gaussian kernel to extract blood vessels while the latter applies a neural network to detect venous beading among the extracted blood vessels for diagnosis (Yang et al., 1995). Proposed a method to detect proliferative retinopathy, Fluorescein-labeled retinal blood vessels were automatically segmented using the Gabor wavelet transform and classified using traditional features such as area, perimeter (Jelinek et al., 2002). This study proposes a way to detect the proliferative diabetic retinopathy on 
the Optic Disc. Since this study focuses on the optic disc, first the segmentation of the optic disc is done by texture descriptors. In Section 2 discuss about the methods used for optic disc segmentation, feature extortion and classification. Section 3 gives the results and Section 4 provides the conclusion and future enhancement.

\section{MATERIALS AND METHODS}

\subsection{Optic Disc Segmentation Using Texture Descriptors}

Generally optic disc is the starting point for the blood vessels, the damage of the new blood vessels on the optic disc will leads in a sever vision loss. In order to segment an image according to its texture, we can measure the texture in a chosen region and then classify it. This is equivalent to template convolution but where the result applied to pixels is the class to which they belong, as opposed to the usual result of template convolution. Many segmentation methods have been already proposed on the segmentation of the optic disc. Initial attempts have been made with shape-based template matching in which OD is modeled as a circular (Lalonde et al., 2001; Chrastek et al., 2002) or elliptical (Abdel-Ghafar and Morris, 2007) object. This matching is performed on an edge map extracted from the underlying image. This approach suffers due to vessel edges present in and around the OD region. To overcome this problem, texture descriptors have been used for the optic disc segmentation. Texture descriptors are used to describe areas within an image where different textures occur. If we use all the elements of I (image), it will slow done the computing process. Instead, texture descriptors that capture the essence of the texture sub-image. If the image, $I$ is in size $M \times M$, it is the divided into subimages of wxw where $\mathrm{w}$ is very much lesser than the M. After sub divides the image, descriptors for each sub-image must be calculated. The result of this is stored by descriptor matrix $\mathrm{D}$ of size $\mathrm{R} \times \mathrm{R}$ where $\mathrm{R}=$ $M / w$. The element of $D$ is a vector $\left[d_{1} d_{2} \ldots d_{n}\right]^{T}$ whose size $\mathrm{n}$ is equal to the number of descriptors. Texture information may be capture through texture descriptors. The purpose of texture description is to derive some measurements that can be used to classify a particular texture. In this study three texture descriptors such as Entropy, Inertia and Energy were used to extract the values. Three descriptors are explained here.

\section{Descriptor 1: Entropy}

This descriptor is a measure of randomness present in the neighborhood of current feature. A high value of $\mathrm{d} 1$ indicates that all elements of I are equal Equation 1:

$$
d_{1}=-\sum_{i=1}^{w} \sum_{j=1}^{w}(i, j) \log I(i, j)
$$

\section{Descriptor 2: Inertia}

Element difference moment of order k, this descriptor has relatively low value when the high values of $\mathrm{Y}$ are near the main diagonal, because the difference ( $i-j)$ is smaller there. Here we have to select a proper value for $\mathrm{k}$ Equation 2:

$d_{2}=-\sum_{i=1}^{w} \sum_{i=1}^{w}(i-j)^{K} I(i, j)$

\section{Descriptor 3: Energy}

This is also called as uniformity. It measures the similarity. It results in a lowest value when all the elements of I are equal Equation 3:

$d_{3}=-\sum_{i=1}^{w} \sum_{j=1}^{w} I(i, j)^{2}$

In the computation of the above descriptors, the indices $i$ and $j$ lie in the overlapping window in $I$, (i.e.,) $(i, j) \in w x w$.

\subsection{Segmentation of Blood Vessels Using Ridge Strength and Watershed Transformation}

Generally, gray scale image processing is simple than the color image. Though the processing of color image is difficult, but color images are provide exact results. Color images are having three color planes namely Red, Green and Blue. The green color plane shows the best contrast between the vessel and the background retina. So the green color plane of the image is chosen. After getting the green plane values, the blood vessel is segmented using Ridge strength and Watershed Transformation.

\subsection{Ridge Strength}

Ridges are defined as points where the image has an extrema in the direction of the largest surface curvature. The ridge strength can be calculated by the dark ridges that are formed by the vessel center lines. Ridge strength $\mathrm{k}$, is given by Equation 4 (Lindeberg, 1994): 


$$
K=\frac{L_{x}^{2} L_{y y}+L_{y}^{2} L_{x x}-2 L_{x} L_{y} L_{x y}}{\left(L_{x}^{2}+L_{y}^{2}\right)^{3 / 2}}
$$

where, $\mathrm{L}$ represents the Gaussian filtered image, $\mathrm{L}_{\mathrm{x}}$ and $\mathrm{L}_{\mathrm{y}}$ represents the first partial derivative of $\mathrm{L}$ with respect to $\mathrm{x}$ and $\mathrm{y}$ and $\mathrm{L}_{\mathrm{xx}}, \mathrm{L}_{\mathrm{yy}}, \mathrm{L}_{\mathrm{xy}}, \mathrm{L}_{\mathrm{yx}}$ represents the second partial derivative of $\mathrm{L}_{\mathrm{x}}$ and $\mathrm{L}_{\mathrm{y}}$. The value of the ridge strength $\mathrm{k}$ is positive for the vessel ridges, negative for areas between the vessels and undefined for the areas where the gradient in both $\mathrm{x}$ and $\mathrm{y}$ direction is zero. Applying an empirically derived threshold, $\mathrm{k}^{\text {th }}$ res is used for the segmentation of the blood vessels. The above method produces a large number of disjoint vessels.

\subsection{Watershed Transformation}

Watershed Transformation is the morphological region based segmentation method based on the topology of the image (Meyer, 1994). The grey image forms the topographic surface. Watershed Transformation divides the image into regions based on the image grey level. The dividing lines are called the Watershed lines and the grouped regions are called the catchment basins. The grey level is inverted such that the blood vessels form the watershed lines. The inverted grey image is filtered with the Gaussian filter such that over-segmentation can be avoided. The watershed regions are calculated using Meyer's algorithm as:

Step 1: A set of markers, pixels where the flooding shall start, are chosen.

Step 2: The neighboring pixels of each marked area are inserted into a priority queue with a priority level corresponding to the grey level of the pixel.

Step 3: The pixel with the highest priority level is extracted from the priority queue. If the neighbors of the extracted pixel that have already been labeled all have the same label, then the pixel is labeled with their label. All non-marked neighbors that are not yet in the priority queue are put into the priority queue.

Step 4: Redo step 3 until the priority queue is empty.

The Watershed Transformation produces closed regions connected by the watershed lines. To remove the non-vessel segments the mean value of ' $k$ ' along each candidate segment is calculated and candidates with mean values less than $\mathrm{k}^{\text {th }}$ results are discarded.

\subsection{Feature Extraction}

In medical imaging, segmentation is important for feature extraction, image measurements and image display. In some applications it may be useful to classify image pixels into anatomical regions, such as bones, muscles and blood vessels, while in others into pathological regions, such as cancer, tissue deformities and multiple sclerosis lesions. In some studies the goal is to divide the entire image into sub regions such as the white matter, gray matter and cerebrospinal fluid spaces of the brain while in others one specific structure has to be extracted, for example breast tumors from magnetic resonance images.

Here Fifteen features were calculated for each segment, based on characteristics human observers use to recognize abnormal vessels. The vessel origin was estimated as follows. Firstly, a median filter was applied to remove smaller vessels. Next a threshold was applied to select the darkest $20 \%$ of pixels, which were assumed to belong to the major blood vessels. The centroid of the result was taken as the approximate origin of the major vessels. The following features were calculated for each segment:

- Segment length: The length of each blood vessel from the origin calculated in pixel

- Gradient: The gradient magnitude of the image at each point gives the direction of the largest possible change in the intensity of the grey image. The gradient is calculated using Sobel gradient operator as Equation 5:

$\mathrm{G}=\sqrt{\mathrm{Gx}^{2}+\mathrm{Gy}^{2}}$

where, $G_{x}, G_{y}$ represents the convolution of original image with the kernel. The mean of the gradient is calculated:

- Gradient Variation: The standard deviation of the Sobel gradient is calculated. This feature is based on the observation that the abnormal vessels have more contrast variation than the normal vessels

- Direction: The angle between a tangent to the segment center point and a line from its center point to the vessel origin. The feature is based on the observation that normal vessels tend to radiate from the vessel origin towards the edge of the disc, whereas the direction of new vessels is more random

- Tortuosity Measure 1: The sum of the absolute changes in the tangential direction along segment path: Equation 6

$\mathrm{T} 1=\frac{1}{\mathrm{n}-1} \sum_{\mathrm{i}=1}^{\mathrm{n}-1}|\theta \mathrm{i}+1-\theta \mathrm{i}|$

where, $\theta \mathrm{i}$ is the tangential angle at the ith element. 
- Tortuosity Measure 2: The difference in the angular extrema of the segment tangents Equation 7:

$\mathrm{T} 2=\max \mathrm{i}=1 \ldots \mathrm{n}\{\theta \mathrm{i}\}-\min \mathrm{i}=1 \ldots . \mathrm{n}\{\theta \mathrm{i}\}$

- Tortuosity Measure 3: The third tortuosity measure was the mean change in direction per pixel along the segment Equation 8 and 9:

$\mathrm{T}_{3}=\frac{1}{\mathrm{n}} \sum_{\mathrm{i}=1}^{\mathrm{n}} \mathrm{ki}$

Where:

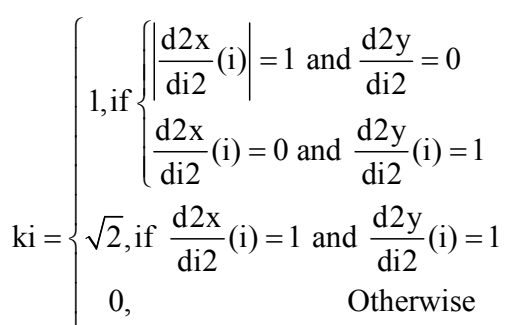

where, $\mathrm{x}$ and $\mathrm{y}$ are the Cartesian coordinates.

The above three tortuosity feature is based on the observation that abnormal vessels are more twisted than the normal vessels.

\subsection{Grey Level}

The normalized mean grey level Equation 10:

$$
g_{\text {norm }}=\frac{1}{G \max -G \min }\left[\left(\frac{1}{n} \sum_{i-1}^{n} g i\right)-G \min \right]
$$

where, gi is the grey level of the ith segment pixel and $G_{\max }$ and $G_{\min }$ are the maximum and minimum grey level values in the original image:

- Grey Level Coefficient of variation: The ratio of the mean and standard deviation of the segment grey level values. This feature is based on the observation that the abnormal vessels have greater variation of grey level than abnormal blood vessels

- Distance from origin: The distance from the center of the segment to the vessel origin in pixel. This feature is based on the observation that the abnormal vessels occur towards the edge of the disc
- Vessel Density: The segment surrounding the segment ' $a$ ' were determined using Equation 11:

$$
\mathrm{O}(\mathrm{a})=\{\mathrm{b} \mid \mathrm{s}(\mathrm{a}) \oplus \mathrm{D}) \cap \mathrm{s}(\mathrm{b}) \neq \varnothing
$$

where, $b \in S$ and $b \neq a$.

where, $S$ is the set of all segments and $s(a) \oplus D$ denotes the dilation between the path of segment ' $a$ ' and structuring element D. The density was taken as the number of elements in $\mathrm{O}$ (a) divided by the length of the segment 'a':

- Number of Segments: The total number of segments following the candidate segmentation. This feature is based on the idea that abnormal vessels have more number of segments than normal vessels

- Mean Ridge Strength: The value of $\mathrm{k}$ by the Equation (4)

- Mean Vessel width: The distance from each segment point to the closest edge point is assumed to be the vessel half-width at that point. This feature is based on the observation that the abnormal vessels are normally very fragile and so the vessel width will be smaller than abnormal vessel

- Mean Vessel wall gradient: The mean value of the Sobel gradient magnitude for all the vessel wall points

\subsection{Classification}

Texture classification process involves two phases: the learning phase and the recognition phase. In the learning phase, the target is to build a model for the image content of each class present in the training data, which generally comprises of images with known class labels. The image content of the training images is captured with the chosen image analysis method, which yields a set of features for each image. These features, which can be scalar numbers or discrete histograms or empirical distributions, characterize given textural properties of the images, such as spatial structure, contrast, roughness, orientation. In the recognition phase the image content of the unknown sample is first described with the same texture analysis method. Then the features of the sample are compared to those of the training images with a classification algorithm and the sample is assigned to the category with the best match. Overall classification and segmentation process in shown in Fig. 2.

There are many segmentation techniques used in multimodality images. Some of them are k-Nearest Neighbors (kNN), k-means, fuzzy c-means, artificial networks algorithms, expectation/maximization and SVM. 


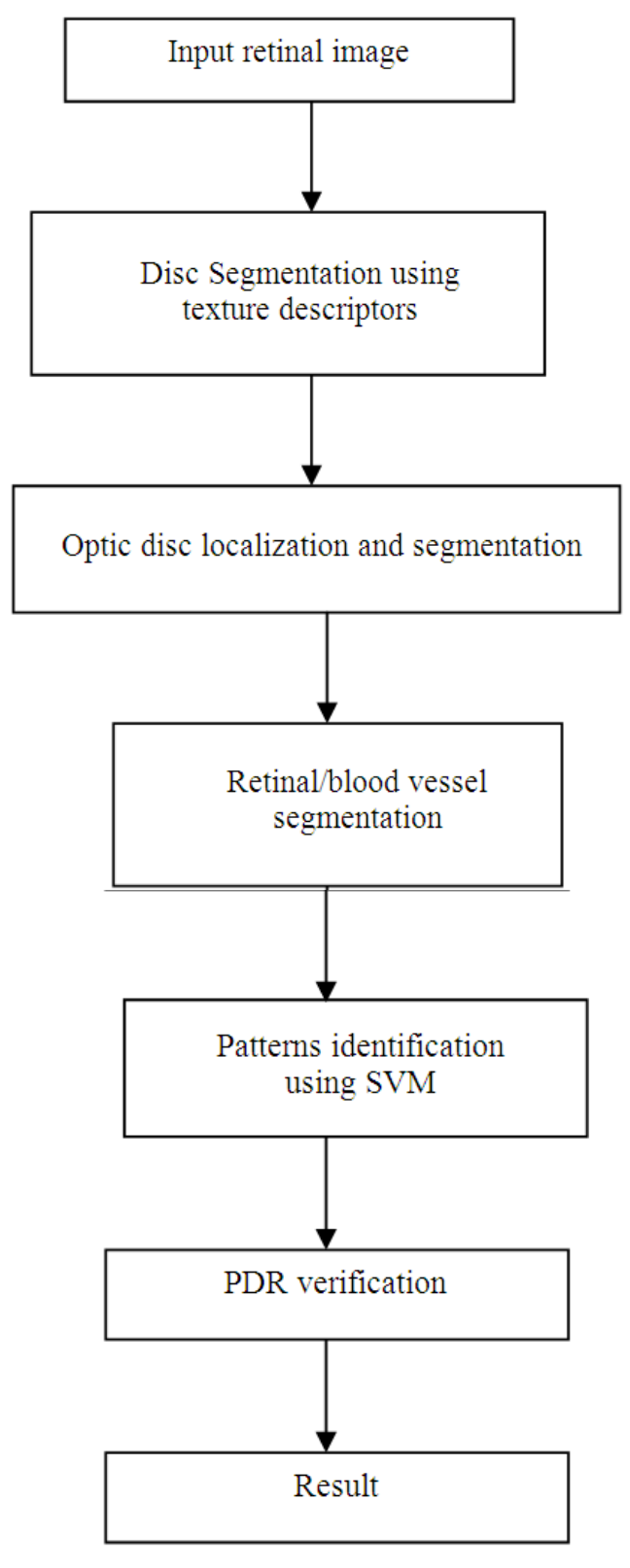

Fig. 2. Over all PDR identification scheme

Support Vector Machines (SVM's) are a relatively new learning method used for binary classification. The basic idea is to find a hyperplane which separates the ddimensional data perfectly into its two classes. However, since example data is often not linearly separable, SVM's introduce the notion of a kernel induced feature space" which casts the data into a higher dimensional space where the data is separable. Typically, casting into such a space would cause problems computationally and with over fitting. The key insight used in SVM's is that the higher-dimensional space doesn't need to be dealt with directly (as it turns out, only the formula for the dotproduct in that space is needed), which eliminates the above concerns. Furthermore, the VC-dimension (a measure of a system's likelihood to perform well on unseen data) of SVM's can be explicitly calculated, unlike other learning methods like neural networks, for which there is no measure. Overall, SVM's are intuitive, theoretically well-founded and have shown to be practically successful. SVM's have also been extended to solve regression tasks (where the system is trained to output a numerical value, rather than yes/no" classification). Support Vector Machine is used as the classifier, for its good classification performance. Each instance in the training set contains one target value (i.e., the class labels) and several attributes (i.e., the features or observed variables). The goal of SVM is to produce a model (based on the training data) which predicts the target values of the test data given only the test data attributes. The training vectors are mapped into a higher dimensional space for classification. The transformation to the higher dimensional space involves the kernel function. SVM finds a hyperplane with the maximal margin in the higher dimensional space. The kernel function used to transform the original feature space to transformed feature space is the radial basis function Equation 12 (Boser et al., 1992; Vapnik, 1998; Wu et al., 2004):

$\mathrm{K}\left(\mathrm{x}_{\mathrm{i}}, \mathrm{x}_{\mathrm{j}}\right)=\exp \left(-\gamma\left\|\mathrm{x}_{\mathrm{i}}-\mathrm{x}_{\mathrm{j}}\right\|^{2}\right) \gamma>0$

where, $\mathrm{x}_{\mathrm{i}}, \mathrm{x}_{\mathrm{j}}$ are the feature vectors for the two classes and $\gamma$ is a configurable parameter.

\subsection{Training}

The retinal image containing the optic disc is first segmented using texture descriptors and the blood vessels are extracted by the Watershed transformation and fifteen features corresponding to the shape and position of the blood vessels are extracted and the features are saved. This training process comprises of training in the normal and abnormal images and the features are saved separately for normal and abnormal images.

\subsection{Testing}

The image to be tested is given as an input and the segmentation of the optic disc is done. The blood vessels are extracted by the Watershed Transformation and 
fifteen features corresponding to each of the blood vessels are extracted and given as an input to the SVM classifier. Based on the training data, the test image features are classified as normal or abnormal.

\section{RESULTS AND DISCUSSION}

The above work is carried out with the dataset of 32 images, out of which 23 are normal images and 9 are abnormal images. Figure 3 and 4 shows the normal and abnormal images respectively. Figure 5 and $\mathbf{6}$ shows the output of various disc detection processes. The image that are actually normal image and classified as normal are called True negative and if the same image is classified as abnormal, it is said to be False positive. The image that is actually abnormal and classified as normal are called false negative and if the same image is classified as abnormal, it is said to be True negative. The accuracy is calculated in terms of sensitivity and specificity as Equation 13 and 14:

$$
\begin{aligned}
& \text { Sensitivity }=\frac{\text { No.of True positive }}{\text { No.of True positive }+ \text { No. of False Negative }} \\
& \text { Specificity }=\frac{\text { No.of Trur Negative }}{\text { No.of True Negative }+ \text { No.of Falsepositive }}
\end{aligned}
$$

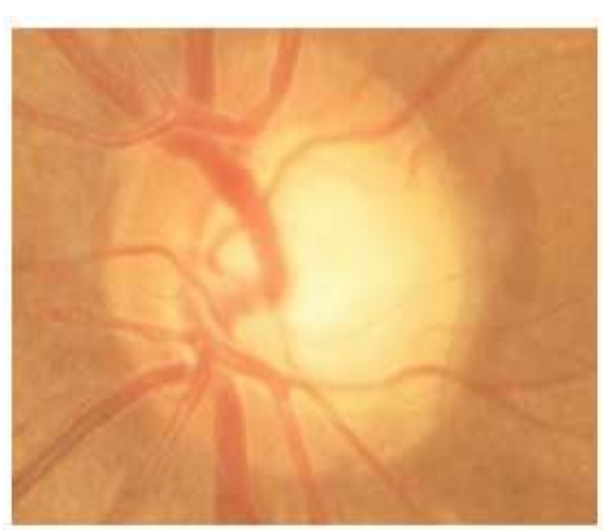

(a)

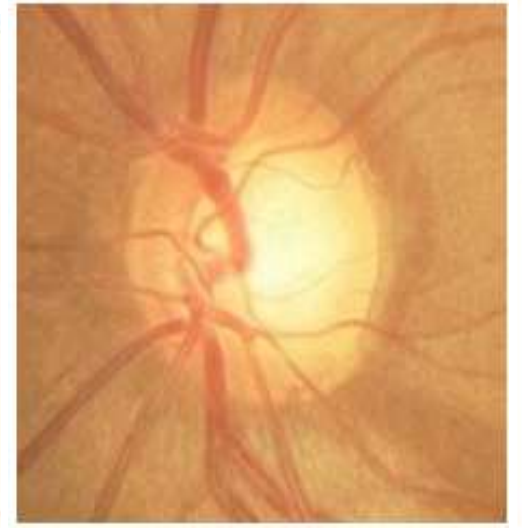

(b)

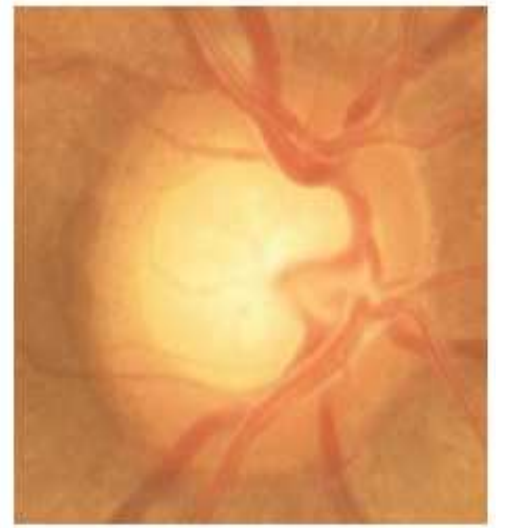

(c)

Fig. 3. Sample normal image

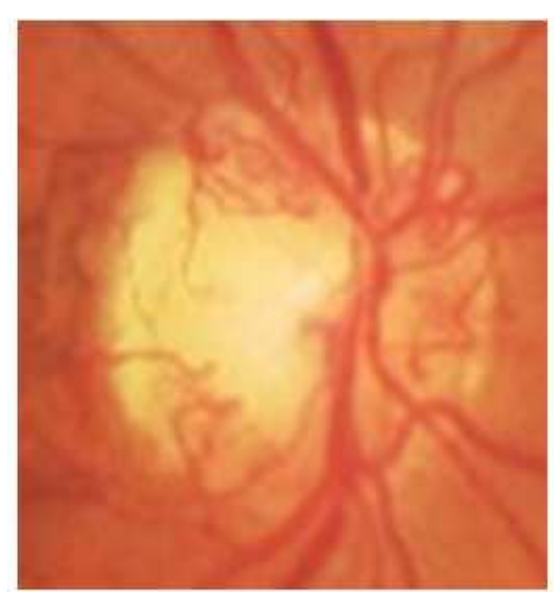

(a)

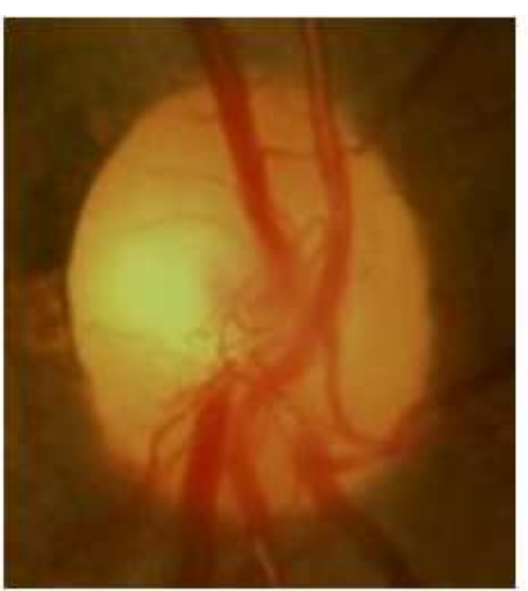

(b)

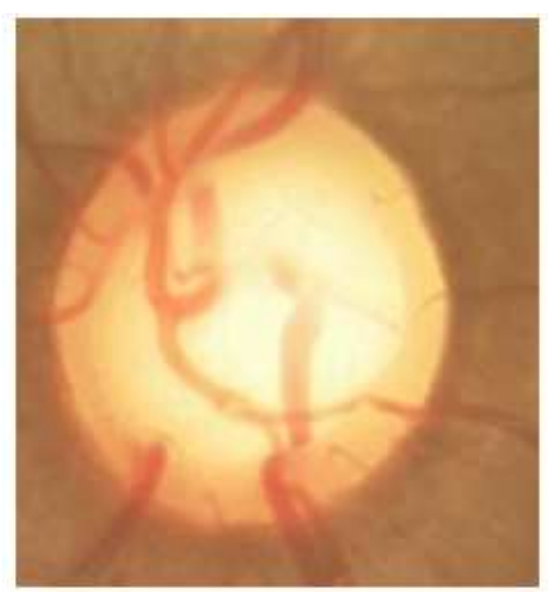

(c)

Fig. 4. Abnormal image 
Tamil Pavai, G. and S. Tamil Selvi / Journal of Computer Science 9 (3): 358-367, 2013

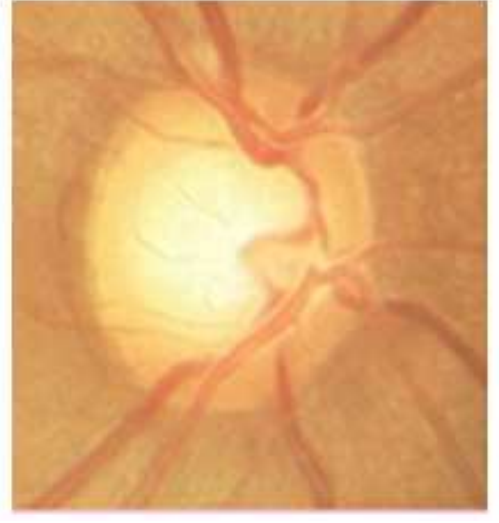

(a)

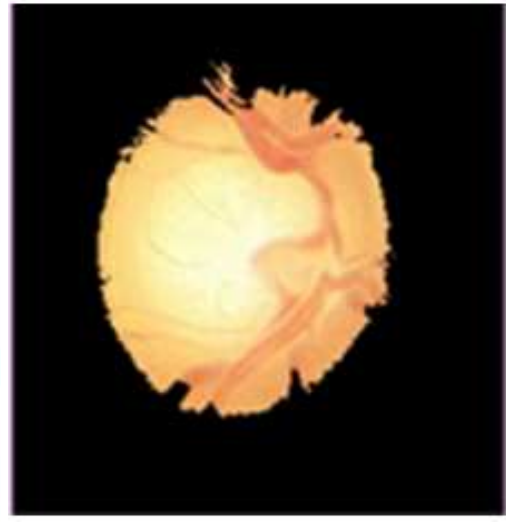

(b)

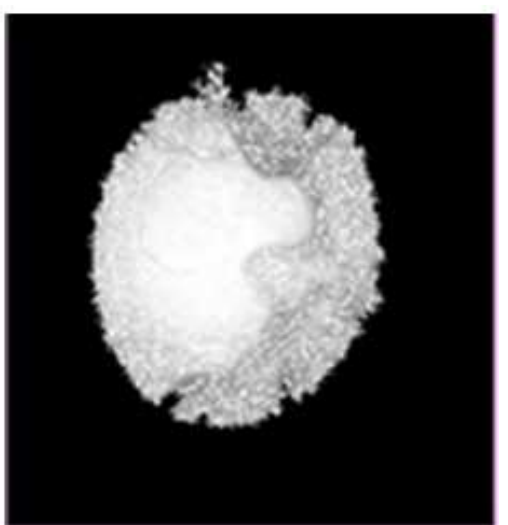

(c)

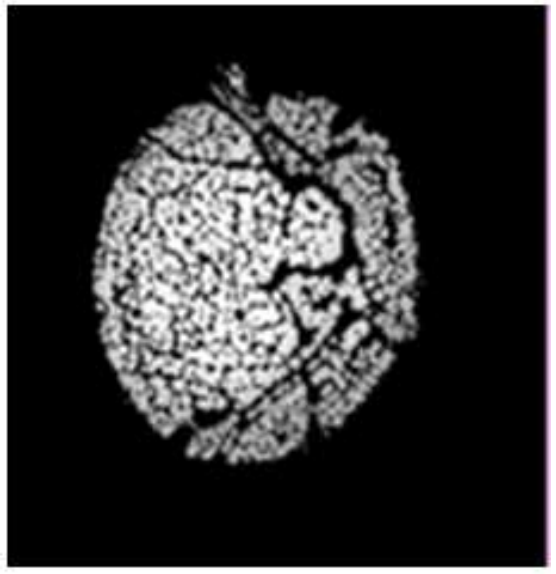

(d)

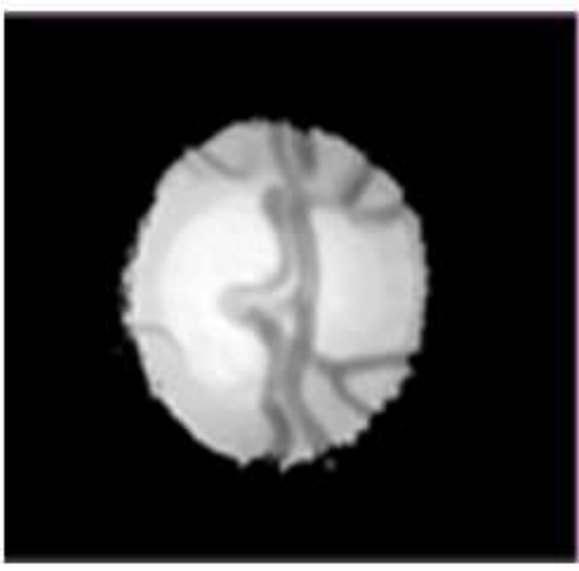

(e)

Fig. 5. (a) Input image segmented, (b) Optic Disc Segmented (c) Ridge Strength Segmented (d) WaterShed (e) Normal Image

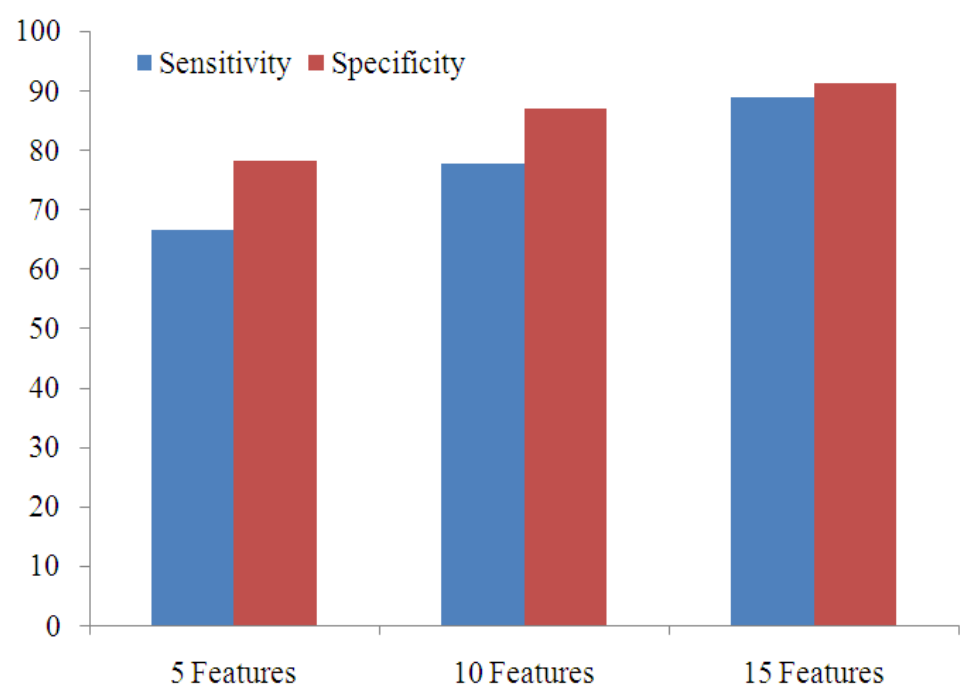

Fig. 6. Graphical representation of sensitivity and specificity 
Table 1. Results for clinical PDR images

\begin{tabular}{lllllll}
\hline No of features & True positive & True negative & False positive & False negative & Sensitivity $(\%)$ & Specificity (\%) \\
\hline 15 & 8 & 21 & 2 & 1 & 88.89 & 91.30 \\
10 & 7 & 20 & 3 & 2 & 77.78 & 86.96 \\
5 & 6 & 18 & 5 & 3 & 66.67 & 78.76 \\
\hline
\end{tabular}

The images are classified as normal or abnormal by as the following table shows the sensitivity and specificity obtained by considering features. The system obtained results are shown in Table 1. The obtained accuracy is sufficient to perform the clinical role and to decrease the workload of an ophthalmologist.

\section{CONCLUSION}

The described method to detect the proliferative diabetic retinopathy shows the sensitivity and specificity of 88.89 and $91.3 \%$. Compare to previous results the system shows higher accuracy when detecting the optic disc by texture descriptors. Since this study detects the disease only on the optic disc, further steps shall be taken to detect the new vessels on the retina. The use of other classifiers such as kNN classifier and the combination of two or more classifiers may also improve the classification performance. Furthermore, the combination of the proliferative diabetic retinopathy detection system with the additional image analysis tool such as non proliferative diabetic retinopathy detection system will prevent the vision loss in the diabetic patient.

\section{REFERENCES}

Abdel-Ghafar, R.A. and T. Morris, 2007. Progress towards automated detection and characterization of the optic disc in glaucoma and diabetic retinopathy. Med. Inform. Internet Med., 32: 19-25. DOI: 10.1080/14639230601095865

Boser, B.E., I.M. Guyon and V.N. Vapnik, 1992. A training algorithm for optimal margin classifiers. Proceedings of the 5th Annual Workshop on Computational Learning Theory, Jul. 27-29, ACM Press, New York, USA., pp: 144-152. DOI: 10.1145/130385.130401

Chen, C.C.H., L.L.F. Pau, P.S.P. Wang and P.S.P. Wang, 1999. The Handbook of Pattern Recognition and Computer Vision. 2nd Edn., World Scientific Publishing Company, Singapore, ISBN-10: 9810230710, pp: 1019.
Chrastek, R., M. Wolf, K. Donath, G. Michelson and H. Niemann, 2002. Optic disc segmentation in retinal images. Proceedings des Workshops Vom Algorithmen Systeme Anwendungen, Mar. 10-12, Springer Berlin Heidelberg, Leipzig, pp: 263-266. DOI: 10.1007/978-3-642-55983-9_60

Hipwell, J.H., F. Strachan, J.A. Olson, K.C. McHardy and P.F. Sharp et al., 2000. Automated detection of microaneurysms in digital red-free photographs: A diabetic retinopathy screening tool. Diabet Med., 17: 588-94. DOI: 10.1046/j.1464-5491.2000.00338.x

Jelinek, H.F., M.J. Cree, J.J. Leandro, J.V.B. Soares and R.M. Cesar et al., 2002. Automated segmentation of retinal blood vessels and identification of proliferative diabetic retinopathy. J. Opt. Soc. Am. A, 24: 1448-56. DOI: 10.1364/JOSAA.24.001448

Lalonde, M., M. Beaulieu and L. Gagnon, 2001. Fast and robust optic disc detection using pyramidal decomposition and Hausdorff-based template matching. IEEE Trans. Med. Imag., 20: 1193-200. DOI: $10.1109 / 42.963823$

Lindeberg, T., 1994. Scale-space theory: A basic tool for analyzing structures at different scales. J. Applied Stat., 21: 225-270. DOI: 10.1080/757582976

Meyer, F., 1994. Topographic distance and watershed lines. Signal Proc., 38: 113-25. DOI: 10.1016/01651684(94)90060-4

Osareh, A., M. Mirmehdi, B. Thomas and R. Markham, 2003. Automated identification of diabetic retinal exudates in digital colour images. Br J. Ophthalmol., 87: 1220-1223. DOI: 10.1136/bjo.87.10.1220

Vapnik, V.N., 1998. Statistical Learning Theory. 1st Edn., Wiley, New York, ISBN-10: 0471030031, pp: 736.

Walter, T., J.C. Klein, P. Massin and A. Erginay, 2002. A contribution of image processing to the diagnosis of diabetic retinopathy-detection of exudates in color fundus images of the human retina. IEEE Trans. Med. Imag., 21: 1236-43. DOI: 10.1109/TMI.2002.806290 
Walter, T., P. Massin, A. Erginay, R. Ordonez and C. Jeulin et al., 2007. Automatic detection of microaneurysms in color fundus images. Med. Image Anal., 11: 555-66. DOI: 10.1016/j.media.2007.05.001

Wu, T.F., C.J. Lin and R.C. Weng, 2004. Probability estimates for multi-class classification by pairwise coupling. J. Mach. Learn. Res., 5: 975-1005. http://dl.acm.org/citation.cfm?id=1016791
Yang, C.W., D.J. Ma, S.C. Chao, C.M. Wang and C.H. Wen et al., 1995. Computer-aided diagnostic detection system of venous beading in retinal images. Opt. Eng., 39: 1293-1303. DOI: 10.1117/1.602487 